\title{
WATER-BLASTING AVOCADOS TO REMOVE LEAFROLLER EGGS
}

\author{
L.E. JAMIESON ${ }^{1}$, D.C. WHITING ${ }^{1}$, A.B. WOOLF ${ }^{1}$ \\ A. WHITE ${ }^{1}$ and R.M. McDONALD ${ }^{2}$ \\ ${ }^{1}$ HortResearch, Private Bag 92169, Auckland, New Zealand \\ ${ }^{2}$ HortResearch, Private Bag 3123, Hamilton
}

\begin{abstract}
The potential for high-pressure water-blasting to remove leafroller egg rafts from the surface of 'Hass' avocados after harvest was investigated. Water pressures from 400 to 1000 psi were tested. Pressures of 800 and 1000 psi removed $96-99.9 \%$ of greenheaded leafroller (Planotortrix octo) egg rafts in two consecutive seasons. Equal numbers of brownheaded leafroller (Ctenopseustis obliquana) egg rafts were also removed at pressures of $800 \mathrm{psi}$. Natural infestations of thrips, mites and leafroller larvae were completely removed at pressures from 400 to 1000 psi but armoured scale pests remained on the fruit. Water-blasted fruit met current industry quality standards.
\end{abstract}

Keywords: greenheaded leafroller, brownheaded leafroller, waterblasting, removal, avocado.

\section{INTRODUCTION}

Australia and USA are important export markets for New Zealand avocados (Persea americana, Mill). If numbers of the native leafrollers, greenheaded leafroller (Planotortrix octo Dugdale) (GLR) and brownheaded leafroller (Ctenopseustis obliquana [Walker]) (BLR) on avocados after harvest exceed the maximum pest limit defined by these countries (Australia, one egg raft per 600 fruit; USA, one egg raft per 1000 fruit) the consignment is either refused access or fumigated.

Currently, fruit bound for Australia are dipped in pyrethrum to control avocado pests. However, this does not result in acceptable mortality of egg rafts. Avocado exports to Australia are inspected in New Zealand by the Australian Quarantine Inspection Service prior to export. If the consignment fails inspection, it is fumigated or sold on the local market at reduced financial return to the grower. Fumigation significantly reduces the quality of avocados (Ito and Hamilton 1980). Avocados exported to the USA are inspected for pest contaminants on arrival. If rejected, high losses are incurred because the avocados are already 2-3 weeks old and cannot be redirected to other markets.

High-pressure water-blasting has been investigated for insect removal from other fruit crops, in particular apples (Whiting et al. 1998a), kiwifruit (Whiting et al. 1998b) and citrus (Walker et al. 1996). The method is currently used to clean fresh produce in USA, Israel and South Africa. This paper presents the results of trials evaluating the potential of water-blasting to remove pests from avocados.

\section{Infestation of fruit with egg rafts}

\section{METHODS}

Insecticide free 'Hass' avocados were artificially inoculated with either GLR or BLR eggs. GLR and BLR adults were obtained from colonies maintained at the insect rearing facility at HortResearch, Auckland. Freshly emerged moths (1:1 sex ratio) were mated over a 3 day period at $20^{\circ} \mathrm{C}$ and then confined to oviposit overnight in mesh lined cages ( 25 moth pairs in each cage) containing a single layer of 10 avocados. The following day the avocados, infested with freshly laid eggs, were removed and held at $6-10^{\circ} \mathrm{C}$ until treatment one to five days later. 


\section{Water-blasting equipment}

For trials carried out in 1998 and 1999 an experimental water-blasting unit, constructed by HortResearch, Ruakura, was used. The unit consisted of a moving set of rollers which carried the avocados in a single lane to the spray site. Three rotating metal arms fed the fruit onto rotating soft-cup rollers to be water-blasted and then pushed the fruit off the rollers. The fruit rolled sideways with their long axis horizontal for treatment. Fruit were exposed to three jet fan nozzles, two angled at $45^{\circ}$ on each side of the fruit and one above the fruit. The top nozzle was set to spray slightly behind the two side jets, both to avoid interference between jets, and to aid fruit rotation. Between 1998 and 1999 changes to the experimental unit included installation of safety guards and the centralisation of the gantry (water supply pipe and nozzle mounting) so that the slight difference in the side nozzle distance in 1998 was removed.

For the trial carried out in 2000 a multi-laned commercial prototype water-blasting unit, constructed by Opotiki Packing and Coolstorage Ltd., with similar operational characteristics to the experimental unit was used.

\section{Treatment procedure}

Prior to treatment all avocados were individually numbered and the number of egg rafts on each fruit recorded. They were then packed at random into replicates of either 25 (1998 and 1999) or 100 (2000) fruit. One replicate was passed through the waterblaster (experimental or commercial prototype) at a time. The order of treatments was randomised and four or five replicates of each pressure executed. Each fruit was exposed to a one-second treatment ( 2 fruit rotations). The avocados were re-packed after each run, held at $6-10^{\circ} \mathrm{C}$ overnight and examined the following day.

In 1998, removal of GLR egg rafts at pressures of 400, 800 and 1000 psi was investigated.

In 1999, removal of GLR egg rafts at pressures of 600, 800 and 1000 psi was investigated and in addition, fruit infested with egg rafts of BLR were also exposed to the 800 psi treatment.

In 2000 GLR egg-infested fruit were treated at 900 psi using the commercial prototype machine.

When using the experimental unit (1998 and 1999), if the orientation of an avocado was incorrect (end-on-end instead of sideways rotation) during treatment, it was discarded and a replacement fruit was treated at the end of that run. When using the commercial prototype (2000) incorrectly orientated fruit were not removed and replaced.

\section{Statistical analysis}

The number of egg rafts per fruit were counted before and after treatment and the results analysed using the Generalised Linear Model procedure (Chambers and Hastie 1992) in S-PLUS (Statistical Sciences Inc. 1996). The model assumed the variance was approximated by a Poisson distribution. The fitted values corresponding to the different water pressure or different species were compared to the values for the same fruit before washing. Standard errors are based on fruit to fruit variation of the leafroller egg raft numbers in each treatment.

\section{RESULTS AND DISCUSSION}

The results for treatments over the 3 years $(1998,1999$ and 2000) are shown in Table 1. After treatment, residues of the egg membranes remained on many of the avocados. These did not contain viable eggs since the developing embryo had been removed and therefore they were not a quarantine risk.

\section{8}

The 400 psi treatment resulted in removal of only $78 \%$ viable egg rafts and $56 \%$ of all evidence of egg rafts (viable and egg membrane residues). The efficacy of removal increased with increasing pressure. Pressures of 800 and 1000 psi removed $96-99 \%$ of viable egg rafts and $78-82 \%$ of all evidence of eggs.

It was observed that all thrips, mites and leafroller larvae and webbing previously noted as present on the fruit were absent after water-blasting, however, armoured scale (mainly present at the top of the fruit) remained. Whiting et al. (1998b) found that 
armoured scale was difficult to remove from kiwifruit using water-blasting. In Israel, insecticides are routinely applied to citrus two to three weeks prior to harvest to kill scale. That procedure may enhance scale removal by water-blasting, as dead scale are easier to remove than live scale (Walker et al. 1999).

\section{TABLE 1: Number and \% removal of Greenheaded leafroller (GLR) and Brownheaded leafroller (BLR) egg rafts after water-blasting (mean $\pm \mathrm{SE})$.}

\begin{tabular}{|c|c|c|c|c|c|c|}
\hline \multirow[t]{2}{*}{$\begin{array}{l}\text { Water } \\
\text { blasting } \\
\text { treatment } \\
\text { (psi) }\end{array}$} & \multirow{2}{*}{$\begin{array}{l}\text { No. } \\
\text { reps }\end{array}$} & \multirow{2}{*}{$\begin{array}{c}\text { No. of } \\
\text { egg rafts/fruit } \\
\text { before } \\
\text { treatment }\end{array}$} & \multicolumn{2}{|c|}{$\begin{array}{l}\text { Number of } \\
\text { egg rafts/fruit } \\
\text { after treatment }\end{array}$} & \multicolumn{2}{|c|}{$\%$ removal } \\
\hline & & & Viable & residue & Viable & residue \\
\hline \multicolumn{7}{|c|}{1998 - GLR } \\
\hline 400 & 4 & $4.48 \pm 0.35$ & $0.97 \pm 0.12$ & $1.96 \pm 0.18$ & $78.2 \pm 3$ & $55.8 \pm 4$ \\
\hline 800 & 4 & $4.33 \pm 0.34$ & $0.17 \pm 0.05$ & $0.99 \pm 0.14$ & $96.0 \pm 1$ & $77.5 \pm 3$ \\
\hline 1000 & 5 & $4.86 \pm 0.33$ & $0.06 \pm 0.03$ & $0.88 \pm 0.12$ & $98.6 \pm 1$ & $81.1 \pm 2$ \\
\hline \multicolumn{7}{|c|}{1999 - GLR } \\
\hline 600 & 4 & $8.77 \pm 0.63$ & $0.70 \pm 0.12$ & $2.32 \pm 0.25$ & $92.0 \pm 1.5$ & $73.5 \pm 3.1$ \\
\hline 800 & 5 & $8.21 \pm 0.55$ & $0.15 \pm 0.05$ & $0.93 \pm 0.14$ & $98.1 \pm 0.6$ & $88.7 \pm 1.7$ \\
\hline 1000 & 4 & $8.72 \pm 0.63$ & $0.10 \pm 0.01$ & $0.47 \pm 0.11$ & $99.9 \pm 0.2$ & $94.6 \pm 1.2$ \\
\hline \multicolumn{7}{|c|}{1999 - BLR } \\
\hline 800 & 5 & $4.43 \pm 0.35$ & $0.11 \pm 0.04$ & $0.38 \pm 0.08$ & $97.5 \pm 0.9$ & $91.3 \pm 1.7$ \\
\hline \multicolumn{7}{|c|}{2000 - GLR } \\
\hline 900 & 4 & $3.14 \pm 0.13$ & $0.27 \pm 0.04$ & $1.55 \pm 0.15$ & $91.6 \pm 1.1$ & $50.9 \pm 3.5$ \\
\hline
\end{tabular}

\section{9}

Successful removal of GLR egg rafts using 800 and 1000 psi was confirmed in the second season. Only one viable egg raft remained after treatment with 1000 psi. Waterblasting at $600 \mathrm{psi}$ was more effective at removing GLR egg rafts than the $400 \mathrm{psi}$ treatment testing in 1998. Efficiency of removal of all signs of eggs was significantly higher when compared to equivalent treatments in 1998, suggesting that the changes to the experimental unit between seasons enhanced its action. At $800 \mathrm{psi}$ egg rafts and residues of both GLR and BLR were removed in equal numbers despite the fact that GLR adults deposit a cementing substance over their egg rafts (A.M. Barrington, pers. comm.).

Fruit quality trials in 1998 and 1999 showed that exposure to 900 psi was tolerated by the avocados (A.B. Woolf, unpubl. data), therefore this was the pressure recommended for use in the commercial water-blaster.

2000

At 900 psi the commercial prototype unit removed $92 \%$ viable egg rafts, equivalent to the 600 psi treatment using the experimental unit. Fifty one percent of all signs of eggs were removed, equivalent to a 400 psi treatment with the experimental unit. With this machine the incorrectly orientated fruit were not discarded, which may account for the lower efficiency of the commercial prototype when compared with the experimental unit.

Our results show that water-blasting removes a high proportion of leafroller egg rafts from avocados. The numbers of egg rafts on the trial fruit (3-8 egg rafts per fruit) were artificially high as a result of enforced oviposition. The number of viable egg rafts on commercial fruit is estimated to be less than one per 1000 fruit. Cubic splines fitted to the percent insect removal data for 1999 predicted that $99.4 \%$ removal of leafroller egg rafts would occur at 900 psi. On the basis of that model, the commercial use of 
water blasting would substantially reduce the probability of viable egg rafts remaining on avocados in export consignments. Modifications to the commercial prototype planned for the 2001 season aim to achieve removal efficiency equivalent to that obtained with the experimental unit.

\section{ACKNOWLEDGEMENTS}

This work was carried out in partnership with the Opotiki Packing and Coolstorage Ltd., Far North Packers and the Avocado Industry Council as a Technology New Zealand TBG project. We thank A.M. Barrington for insect supply, P.G. Connolly for statistical analyses of the data, B.T. Boxer for technical assistance, B. Stevenson for engineering assistance and R.A. Fullerton and P.S. Stevens for comments on the manuscript.

\section{REFERENCES}

Chambers, J.M. and Hastie, T.J., 1992. Statistical Models in S. Wadsworth and Brooks/Cole, Pacific Grove, California. 608 pp.

Ito, P.J. and Hamilton R.A.,1980. Fumigation of avocado fruit with methyl bromide. HortSci. 15: 593.

Statistical Sciences Inc., 1996. S-PLUS User's Manual, version 3.2, Statistical Sciences Inc., Seattle, Washington.

Walker, G.P., Morse, J.G. and Arpaia, M.L., 1996. Evaluation of a high-pressure washer for postharvest removal of California red scale (Homoptera: Diapididae) from citrus fruit. J. Econ. Ent. 89: 148-155.

Walker, G.P., Zareh, N. and Arpaia, M.L., 1999. Effect of pressure and dwell time on efficiency of a high-pressure washer for postharvest removal of California red scale (Homoptera: Diapididae) from citrus fruit. J. Econ. Ent. 92: 906-914.

Whiting, D.C., Hoy, L.E., Maindonald, J.H., Connolly P.G. and McDonald, R.M., 1998a. High-pressure washing treatments to remove obscure mealybug (Homoptera: Pseudococcidae) and lightbrown apple moth (Lepidoptera: Tortricidae) from harvested apples. J. Econ. Ent. 91: 1458-1463.

Whiting, D.C., Hoy, L.E., Connolly, P.G. and McDonald, R.M., 1998b. Effects of high-pressure water jets on armoured scale insects and other contaminants of harvested kiwifruit. Proc. 51st N.Z. Plant Prot. Conf.: 211-215. 